\title{
Maximum Entropy Modeling with Clausal Constraints*
}

\author{
Luc Dehaspe
}

\author{
Department of Computer Science, Katholieke \\ Universiteit Leuven \\ Celestijnenlaan 200A, B-3001 Heverlee, Belgium \\ email : Luc.Dehaspe@cs.kuleuven.ac.be \\ telephone : ++3216327567 ; fax : ++3216327996
}

\begin{abstract}
We present the learning system MACCENT which addresses the novel task of stochastic MAximum ENTropy modeling with Clausal Constraints. Maximum Entropy method is a Bayesian method based on the principle that the target stochastic model should be as uniform as possible, subject to known constraints. MACCENT incorporates clausal constraints that are based on the evaluation of Prolog clauses in examples represented as Prolog programs. We build on an existing maximum-likelihood approach to maximum entropy modeling, which we upgrade along two dimensions: (1) MACCENT can handle larger search spaces, due to a partial ordering defined on the space of clausal constraints, and (2) uses a richer firstorder logic format. In comparison with other inductive logic programming systems, MACCENT seems to be the first that explicitly constructs a conditional probability distribution $p(C \mid I)$ based on an empirical distribution $\tilde{p}(C \mid I)$ (where $p(C \mid I)(\tilde{p}(C \mid I))$ gives the induced (observed) probability of an instance $I$ belonging to a class $C$ ). First experiments indicate MACCENT may be useful for prediction, and for classification in cases where the induced model should be combined with other stochastic information sources.
\end{abstract}

\footnotetext{
*Also submitted to the Seventh International Workshop on ILP (ILP'97).
}

\section{Introduction}

Bayesian probability theory permits one to use prior knowledge, in the form of an empirical probability distribution, for making rational inferences about the behavior of a random process. Maximum Entropy [Jaynes, 1990] method is a Bayesian method based on the principle that the target stochastic model should be as uniform as possible, subject to constraints extracted from the training distribution. This principle is not new, it can in fact be traced back to Occam's razor, Laplace, and beyond, but relatively recent developments in computer science have boosted its popularity and have enabled its application to real-world problems, mainly in physics (cf. [Gull and Daniell, 1978]). As a starting point for our research we used the paper by Berger, Della Pietra, and Della Pietra [Berger et al., 1996], which presents a maximum-likelihood approach to the construction of maximum entropy models of natural language.

We have combined the statistical approach of [Berger et al., 1996] with techniques from the field of inductive logic programming to build the relational learner MACCENT that addresses the task of MAximum ENTropy modeling with Clausal Constraints. The adjective "clausal" here refers to the fact that in MACCENT the constraints, which are the building blocks of maximum entropy modeling, are based on the evaluation of Prolog clauses in examples represented as Prolog programs. The integration of probabilistic methods with inductive logic programming has recently become a popular research topic (cf. [Karalič and Bratko, 1997; Muggleton, 1997; Pompe and Kononenko, 1995]), but, to the best of our knowledge, MACCENT is the first inductive logic programming algorithm that explicitly constructs a conditional probability distribution $p(C \mid I)$ based on an empirical distribution $\tilde{p}(C \mid I)$ (where $p(C \mid I)$ $(\tilde{p}(C \mid I))$ gives the induced (observed) probability of an instance $I$ belonging to a class $C$ ).

We start our discussion with a brief introduction to the logical and mathematical foundations (Section 2). In the two next parts we then present the task addressed by 


\begin{tabular}{|c|c|c|c|c|c|}
\hline animal & has_covering & has_legs & habitat & homeothermic & class \\
\hline dog & hair & yes & land & yes & mammal \\
dolphin & none & no & water & yes & mammal \\
trout & scales & no & water & no & fish \\
shark & none & no & water & no & fish \\
herring & scales & no & water & no & fish \\
eagle & feathers & yes & air & yes & bird \\
penguin & feathers & yes & water & yes & bird \\
lizard & scales & yes & land & no & reptile \\
snake & scales & no & land & no & reptile \\
turtle & scales & yes & land & no & reptile \\
\hline
\end{tabular}

from interpretations paradigm, introduced by [De Raedt and Džeroski, 1994] and related to other inductive logic programming settings in [De Raedt, 1996].

\subsection{Statistical paradigm: maximum entropy}

Table 1: The animals domain

MACCENT (Section 3), and the algorithm itself (Section 4). Finally, we report on classification and prediction experiments with a prototypical implementation of MACCENT (Section 5), and conclude (Section 6). Though throughout the paper we have restricted the logical notation to a minimum, we do assume some familiarity with the programming language Prolog [Bratko, 1986].

\section{Logical and statistical background}

\subsection{Logical paradigm: learning from interpretations}

Consider the data in Table 1, which presents instances of four classes of animals. Traditional attribute-value learners represent each animal and candidate classification rule as a set of attribute-value pairs, such that simple subset test suffices in order to verify if a rule covers an instance. Alternatively, one can store the information available about a single animal in a Prolog knowledge base, encode candidate rules as Prolog queries, and apply the Prolog execution mechanism to verify whether or not a rule covers an instance. If the query succeeds, the rule covers the instance. If the query fails ${ }^{1}$, the rule does not cover the instance. For example, the logical conjunction has_legs $\wedge$ habitat(land) covers instance dog because the Prolog query $\leftarrow$ has_legs, habitat(land) succeeds in the Prolog program dog:

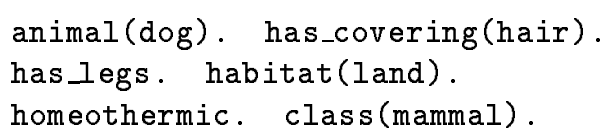

Notice that the attribute-value and Prolog representations are equivalent in standard propositional domains. In Prolog notation however instances can be Prolog programs of deliberate size and complexity, rather than fixed-length vectors of attribute-value pairs. As a consequence, background knowledge can be added in a natural way as Prolog code shared by all instances. For example, with Prolog clause kids_love_it :- homeothermic, habitat (water) added as background knowledge to Table 1 , rule has_covering $($ none $) \wedge k i d s \_l o v e \_i t$ exclusively covers instance dolphin.

The above alternative and flexible Prolog based representation and verification method fits in the learning

${ }^{1}$ Termination can be guaranteed by employing a depthbound on the depth of the proof tree.

Let us reconsider the data in Table 1, and interpret them as as a sample of an expert biologist's decisions concerning the class of animals. The observed behavior of the biologist can be summarized as an empirical conditional distribution $\tilde{p}(C \mid I)$ that, given the description of an instance in $I$ in the first five columns, assigns probability 1 to the class specified in the last column, and 0 to all other classes. Sample $\tilde{p}$ can be used to support the construction of a stochastic model $p$ to predict the behavior of the biologist. Given an instance $I$, this target model will assign to each class $C_{j}$ an estimate $p\left(C_{j} \mid I\right)$ of the conditional probability that the biologist would classify $I$ as $C_{j}$.

"Relevant" facts (statistics) extracted from the empirical distribution $\tilde{p}$ are the building blocks of the target distribution $p$. For example, with sample Table $1, \tilde{p}$ assigns conditional probability 1 to exactly four classes $C$. If we are convinced this fact captures relevant information about the sample, we can construct our model $p$ such that, for all instances $I$ it meets the following constraint:

$p(\operatorname{mammal} \mid I)+p($ fish $\mid I)+p($ bird $\mid I)+p($ reptile $\mid I)=1$

There is of course a whole family of models that meet constraint (1). However, some members of this family seem more justified than others, given the limited empirical evidence (1) we have gathered so far. More specifically, the model that assigns equal 0.25 probability to all classes seems intuitively more reasonable than one that grants probability 0.5 to mammal and fish, and 0 to the other classes. Though the latter model agrees with what we know, it contains information for which we have - as yet - no evidence. A stochastic model that avoids such bold assumptions, should be as "uniform" as possible, subject to the known constraints.

In the maximum entropy paradigm [Jaynes, 1990] conditional entropy

$$
H(p) \equiv-\frac{1}{N} \sum_{C, I} p(C \mid I) \log p(C \mid I)
$$

is used to measure uniformity ${ }^{2}$, with $N$ the number of instances in the sample. The Maximum Entropy Principle then states that, from the family of conditional probability distributions allowed by an empirically compiled set of constraints, we should elect the model with maximum conditional entropy $H(p)$.

\footnotetext{
${ }^{2}$ Throughout the paper log denotes natural logarithm.
} 


\section{Maximum entropy modeling with clausal constraints}

As indicated in the previous section, the building blocks of a maximum entropy model are constraints such as Equation (1). MACCENT incorporates constraints based on boolean clausal indicator functions $f(I, C)$ with general form:

$f_{j}(I, C)=\left\{\begin{array}{ll}1 & \text { if } C=C_{j} \\ 0 & \text { otherwise }\end{array}\right.$ and query $\leftarrow Q_{j}$ succeeds in instance $I$

A clausal ${ }^{3}$ indicator function $f_{j}$, fully defined by the tuple $\left\langle C_{j}, \leftarrow Q_{j}\right\rangle$, gives rise to a clausal constraint of the form

$$
C C_{j}: p\left(f_{j}\right)=\tilde{p}\left(f_{j}\right)
$$

where $p\left(f_{j}\right)$ is the expected value of $f_{j}(I, C)$ in the target distribution, and $\tilde{p}\left(f_{j}\right)$ is the expected value of $f_{j}(I, C)$ in the empirical distribution. For any $f_{j}(I, C)$, the calculation of its expected value with respect to a conditional distribution proceeds as follows. First, we take the sum over all instances and classes $\sum_{I, C} f_{j}(I, C)$. For example, given the data in Table 1, if

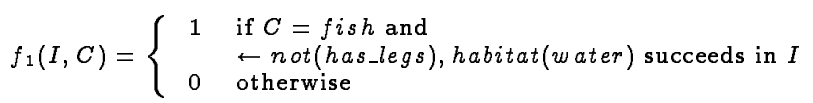

then

$f_{1}($ dolphin, fish $), f_{1}$ (trout, fish), $f_{1}($ shark, fish), and $f_{1}$ (herring, fish) evaluate to one, and all other combinations of instances and classes evaluate to zero, hence $\sum_{I, C} f_{1}(I, C)=4$. Next, we normalize the summation with constant $N$, the number of instances in the sample, for example $1 / 10 \sum_{I, C} f_{1}(I, C)=0.4$. Finally, to obtain the expected value $p\left(f_{j}\right)$ of $f_{j}(I, C)$ in model $p$, we multiply $f_{j}(I, C)$ by $p(C \mid I)$. For the expected value of $f_{j}(I, C)$ in empirical distribution $\tilde{p}$, and in target distribution $p$ this yields the equations

$$
\begin{aligned}
\tilde{p}\left(f_{j}\right) & \equiv \frac{1}{N} \sum_{I, C} \tilde{p}(C \mid I) f_{j}(I, C) \\
p\left(f_{j}\right) & \equiv \frac{1}{N} \sum_{I, C} p(C \mid I) f_{j}(I, C)
\end{aligned}
$$

For example, with $p$ a uniform conditional distribution, $p($ fish $\mid I)=0.25$ for all instances in Table 1 . As $f_{1}(I, f i s h)$ equals one for only four for these, we have that $p\left(f_{1}\right)=1 / 10 *(0.25+0.25+0.25+0.25)=0.1$. On the other hand, in the sample we find that $\tilde{p}($ fish $\mid I)=1$ for the three instances trout, shark, and herring, and $\tilde{p}($ fish $\mid I)=0$ for all other instances, including dolphin. Hence, $\tilde{p}\left(f_{1}\right)=1 / 10 *(1+1+1)=0.3$. Function $f_{1}$, through its expected value, thus reveals a discrepancy between the empirical and the target distribution. This

\footnotetext{
${ }^{3}$ Recall that Prolog queries are a special type of Prolog clauses with false in the conclusion part.
}

discrepancy disappears if we add the clausal constraint $p\left(f_{1}\right)=\tilde{p}\left(f_{1}\right)=0.3$ to our initial constraint Equation (1), and construct a new model that maximizes conditional entropy subject to both constraints. As a second example, consider

$f_{2}(I, C)= \begin{cases}1 & \text { if } C=\text { reptile and } \\ 0 & \begin{array}{c}\leftarrow \text { not(has_covering } \\ \text { otherwise }\end{array}\end{cases}$

for which the expected values are $p\left(f_{2}\right)=0.125$, and $\tilde{p}\left(f_{2}\right)=0.1$. Notice that with respect to the sample the expected value $p\left(f_{2}\right)$ is 0.025 units too high, whereas $p\left(f_{1}\right)$ is 0.2 units too low.

Now that we have described the building blocks, we can formulate the task of maximum entropy modeling with clausal constraints:

- Given: A set $\mathcal{I}$ of $N$ training instances, represented as Prolog programs;

- Given: An empirical conditional distribution $\tilde{p}(C \mid I)$, with $I \in \mathcal{I}$;

- Given: A language $\mathcal{L}$ of clausal constraints $p\left(f_{j}\right)=$ $\tilde{p}\left(f_{j}\right)$;

- Find: An optimal set $\mathcal{C C} \subseteq \mathcal{L}$ of clausal constraints;

- Find: A stochastic model $p^{\mathcal{C C}}(C \mid I)$ that maximizes conditional entropy $H(p)$ subject to the clausal constraints in $\mathcal{C C}$.

To restate, algorithms that address this task receive as input training instances, an empirical conditional distribution that relates training instances to classes, and a set of clausal constraints. In response, they must (1) select an "optimal" subset of clausal constraints, and (2) with these constraints build a model for use in classifying new instances.

We come back to the selection of $\mathcal{C C}$ later, and first concentrate on the modeling subtask. Given set $\mathcal{C C}$, the method of Lagrange multipliers from the theory of constrained optimization produces the model $p$ that maximizes conditional entropy $H(p)$. As discussed thoroughly in [Della Pietra et al., 1995; Berger et al., 1996], the model in question has the parametric form

$$
p_{\Lambda}(C \mid I)=\frac{1}{Z_{\Lambda}(I)} \exp \left(\sum_{j} \lambda_{j} f_{j}(I, C)\right)
$$

where each parameter (Lagrange multipliers) $\lambda_{j}$ from set $\Lambda$ corresponds to a clausal constraint $C C_{j}$ based on clausal indicator function $f_{j}$. The optimal parameter set $\Lambda$ can be determined by maximizing the log-likelihood $L_{\tilde{p}}\left(p_{\Lambda}\right)$ of the empirical distribution $\tilde{p}$, which is defined by

$$
L_{\tilde{p}}\left(p_{\Lambda}\right)=-\frac{1}{N} \sum_{I} \log Z_{\Lambda}(I)+\sum_{j} \lambda_{j} \tilde{p}\left(f_{j}\right)
$$




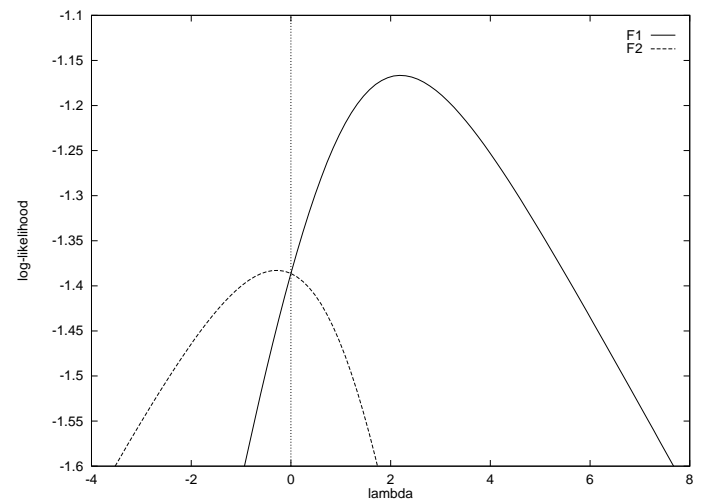

Figure 1: The log-likelihood functions $F 1=L_{\tilde{p}}\left(p_{\left\{\lambda_{1}\right\}}\right)$ and $F 2=L_{\tilde{p}}\left(p_{\left\{\lambda_{2}\right\}}\right)$.

where $Z_{\Lambda}(I)$ is a normalization constant:

$$
Z_{\Lambda}(I)=\sum_{C} \exp \left(\sum_{j} \lambda_{j} f_{j}(I, C)\right)
$$

If we apply these equations to our running example, we obtain, after reduction,

$L_{\tilde{p}}\left(p_{\left\{\lambda_{1}\right\}}\right)=-0.4 * \log \left(e^{\lambda_{1}}+3\right)-0.6 * \log (4)+0.3 * \lambda_{1}$

$L_{\tilde{p}}\left(p_{\left\{\lambda_{2}\right\}}\right)=-0.5 * \log \left(e^{\lambda_{2}}+3\right)-0.5 * \log (4)+0.1 * \lambda_{2}$

where $\lambda_{1}$ and $\lambda_{2}$ correspond to $f_{1}$ and $f_{2}$. The graph of these two equations is plotted in Figure 1 . On the $X$ axis of this graph, we find the $\lambda$ values, on the $Y$ axis the log-likelihood of the Table 1 training set. The graph shows that with $\mathcal{C C}=\left\{C C_{1}\right\}$, the optimal parameter set $\left\{\lambda_{1}\right\}$ is obtained with a positive value for $\lambda_{1}$. This agrees with the earlier finding that in the uniform distribution, $p\left(f_{1}\right)<\tilde{p}\left(f_{2}\right)$. The intuition here is that the appropriateness of $C C_{1}$ has been underestimated in $p$, and a positive weight $\lambda_{1}$ is required to correct this. The opposite holds for $\mathcal{C C}=\left\{C C_{2}\right\}$, where the optimum for $\lambda_{2}$ is negative.

Let us now return to the selection of an optimal set $\mathcal{C C} \subset \mathcal{L}$. For the time being we assume an exhaustive search through $2^{\mathcal{L}}$ and focus on an evaluation function for $\mathcal{S} \subset \mathcal{L}$. Notice that maximum entropy and maximum likelihood are dual problems and that, of all models of parametric form (7), the maximum entropy model is the one that maximizes the the likelihood of the sample. As might be clear from the above discussion of Figure 1, this maximum is function of the set $\mathcal{S}$ of clausal constraints. We are interested in the set $\mathcal{S}$ that produces the highest likelihood of the training sample $\tilde{p}$. Formally, if we let $p^{\mathcal{S}}$ denote the maximum entropy model obtained with a set $\mathcal{S}$ of clausal constraints then

$$
\mathcal{C C} \equiv \underset{\mathcal{S} \subset \mathcal{L}}{\operatorname{argmax}} L_{\tilde{p}}\left(p^{\mathcal{S}}\right)
$$

Algorithm 1 : MACCENT

Inputs: Language $\mathcal{L}$; empirical distribution $\tilde{p}(C \mid I)$ Outputs: $\quad$ Set $\mathcal{C C}$ of clausal constraints; model $p^{\mathcal{C C}}$

1. Start with $\mathcal{C C}=\emptyset$; thus $p^{\mathcal{C C}}$ is uniform

2. Select next clausal constraint $\widehat{c c}$ from $\mathcal{L}$ using Algorithm 2

3. Check the termination condition

4. Adjoin $\widehat{c c}$ to $\mathcal{C C}$

5. Compute $p^{\mathcal{C C}}$ using Equation (7)

6. Go to step 2

For an illustration of this evaluation function consider again Figure 1. The graph shows that with $\left\{C C_{1}\right\}$ we can obtain log-likelihood around -1.17 , whereas with $\left\{C C_{2}\right\}$ we only reach -1.38 . Hence, of these two sets, $\left\{C C_{1}\right\}$ is to be preferred.

\section{The Maccent Algorithm}

In this section we present the learning system MACCENT which addresses the task of MAximum ENTropy modeling with Clausal Constraints. The task as described in the previous section does not directly translate to a tractable algorithm. Usually, with millions of clausal constraints in $\mathcal{L}$, it is prohibitive to inspect all subsets of $\mathcal{L}$ in search for the optimal one. In our description of MACCENT we will gradually abandon the request for optimality of $\mathcal{C C}$. We first discuss a greedy search method and an approximate gain evaluation function, both due to [Berger et al., 1996]. In addition, we then propose a structure on $\mathcal{L}$ that one can use to organize the search process.

As shown in Algorithm 1, MACCENT carries out a greedy search, adjoining clausal constraints one at a time, using an evaluation function to select the next constraint at each step. Given a set $\mathcal{S}$ of constraints collected so far, a natural evaluation function for a candidate clausal constraint $\widehat{c c}$ returns the gain $\triangle L_{\tilde{p}}(\mathcal{S}, \widehat{c c})$ in the log-likelihood of the training data obtained by that adding $\widehat{c c}$ to $\mathcal{S}$

$$
\triangle L_{\tilde{p}}(\mathcal{S}, \widehat{c c}) \equiv L_{\tilde{p}}\left(p^{\mathcal{S} \cup \widehat{c c}}\right)-L_{\tilde{p}}\left(p^{\mathcal{S}}\right)
$$

As $L_{\tilde{p}}\left(p^{\mathcal{S}}\right)$ remains constant, this comes down to adding at each step the constraint $C C$ that maximizes $L_{\tilde{p}}\left(p^{\mathcal{S} \cup C C}\right)$. We have thus reduced the selection task to the discovery of the best $C C \in \mathcal{L}$. For instance, consider again our running example and the graph in Figure 1. Knowing that $L_{\tilde{p}}\left(p^{\emptyset}\right)=-\log (4) \simeq-1.39$, we can compute that the gain obtained by adding $C C_{1}$ and $C C_{2}$ is something round 0.22 and 0.01 respectively. Hence, $C C_{1}$ is a better initial choice. 
Still, given the "old" set $\mathcal{S}=\left\{C C_{1}, \ldots, C C_{n}\right\}$, log-likelihood gain requires for each candidate $\widehat{c c} \in$ $\mathcal{L}$ the computation of the set of parameters $\Lambda=$ $\left\{\lambda_{1}, \ldots, \lambda_{n}, \alpha\right\}$ that maximizes $L_{\tilde{p}}\left(p_{\Lambda}\right)$, where $\alpha$ is the parameter that corresponds to $\widehat{c c}$. Various numerical methods have been applied to this problem, including problem specific algorithms such as Brown [Brown, 1959] or ITERATIVE SCALING [Darroch and Ratcliff, 1972]. However, none of these methods allow for a sufficiently efficient computation of gain $\triangle L_{\tilde{p}}(\mathcal{S}, \widehat{c c})$. As a remedy, [Berger et al., 1996] introduce the notion of approximate gain which no longer requires the computation of $n+1$ parameters. The "old" parameter set $\left\{\lambda_{1}, \ldots, \lambda_{n}\right\}$ is retained, and the approximation is made that the addition of constraint $\widehat{c c}$ affects only parameter $\alpha$. Given candidate constraint $\widehat{c c}$ derived from indicator function $\hat{f}$, Equation (8) then reduces to

$$
G_{\mathcal{S}, \hat{f}}(\alpha)=-\frac{1}{N} \sum_{I, C} \log \left(p^{\mathcal{S}}(C \mid I) e^{\alpha \hat{f}(I, C)}\right)+\alpha \tilde{p}(\hat{f})
$$

Equation (12) in turn simplifies the computation of the approximate gain

$$
\sim \triangle L_{\tilde{p}}(\mathcal{S}, \widehat{c c}) \equiv \max _{\alpha} G_{\mathcal{S}, \hat{f}}(\alpha)
$$

to a one-dimensional optimization problem which can be solved, for instance, with Newton's method.

Though [Berger et al., 1996] has shown one can implement a high-performance algorithm on the basis of the above approximations, we still assume an exhaustive search through a flat, unstructured space $\mathcal{L}$ of clausal constraints. To further speed up the search, and enable the exploration of bigger search spaces, MACCENT extends these approximations with classical machine learning techniques. The key observation here is that generality imposes a partial ordering on $\mathcal{L}$. To be precise, this generalisation relation is defined between tuples of class names and Prolog queries ${ }^{4}:\left\langle C_{g}, \leftarrow Q_{g}\right\rangle$ is more general than $\left\langle C_{s}, \leftarrow Q_{s}\right\rangle$ if and only if $C_{g} \leftarrow Q_{g}$ is logically more general (under $\theta$ subsumption, cf. [Plotkin, 1970]) than $C_{s} \leftarrow Q_{s}$. One can specify a lattice for clausal constraints with a set of most general descriptions $<C_{j}, \leftarrow$ true $>$ at the top, and a specialisation operator for top-down exploration of this lattice. In MACCENT we handle both tasks with the declarative language bias formalism DLAB [Dehaspe and De Raedt, 1996].

As shown in Algorithm 2, MACCENT exploits the lattice structure of $\mathcal{L}$ to carry out a general-to-specific heuristic beam search for clausal constraints with satisfactory, rather than optimal, approximate gain. At each

\footnotetext{
${ }^{4}$ Recall (cf. Equations (3) and (4)) that the tuple $<C_{j}, \leftarrow$ $Q j>$ fully identifies clausal constraint $C C_{j}$.
}

Algorithm 2 : Select next clausal constraint

Inputs: $\quad$ Language $\mathcal{L}$; empirical distribution $\tilde{p}(C \mid I)$; current set $\mathcal{C C}$

Outputs: Clausal constraint $\widehat{c c}$

1. Initialize current best approximate gain Best $:=-1$

2. Initialize Beam $:=\left\{\left\langle C_{j}, \leftarrow\right.\right.$ true $\left.\rangle \mid \operatorname{class} C_{j} \in \mathcal{L}\right\}$

3. Select $c c$ from Beam

4. For each $c c$ in Beam, and for each specialisation $c c^{\prime} \in \mathcal{L}$ of $c c$ :

(a) Compute Gain $=\triangle L_{\tilde{p}}\left(\mathcal{C C}, c c^{\prime}\right)$ using Equation (13),

(b) If Gain > Best then update Best := Gain and $\widehat{c c}:=c c^{\prime}$,

(c) Add $c c^{\prime}$ to Beam

5. Prune Beam

6. If Beam is not empty, go to step 3

level of its search, MACCENT considers all specialisations of clausal constraints in the beam, and if necessary updates the current best solution.

We still have to specify a stopping criterion (Algorithm 1, step 3) and a procedure for pruning Beam (Algorithm 2, step 5). For our stopping criterion we use Equation (8) to calculate the log-likelihood $L_{\bar{p}}\left(p^{\mathcal{C C} \cup \widehat{c c}}\right)$ of a separate empirical distribution $\bar{p}$. If the log-likelihood of this witheld portion of the training set decreases ${ }^{5}$ with the addition of $\widehat{c c}$, this is taken as an indication of overtraining, and MACCENT terminates.

Given a beam size $B S$, the default strategy for pruning Beam is to rank the clausal constraints $c c^{\prime}$ in Beam according to their Gain and keep at most $B S$ of the best scoring constraints. However, in cases where the input empirical distribution assigns only probabilities 1 and 0 , as for instance in Table 1, we can apply a more sophisticated heuristic. Thereto, we define the notion of potential approximate gain as an upper bound to the maximum Gain that can be obtained via further specialisation of $c c^{\prime}$. We cannot go into details here, but under the circumstances mentioned, potential approximate gain can easily be computed. One has to select the instances for which $\tilde{p}\left(C^{\prime} \mid I\right)=1$, compute the approximate gain counting only this subset, do the same for the complementary subset, and take the maximum of both computations. The potential approximate gain upper bound allows a branch-and-bound search in which clausal constraints are pruned that after specialisation can never produce an approximate gain above the current best. Apart from that, potential approximate gain can also be used in combination with actual approximate

\footnotetext{
${ }^{5} \mathrm{~A}$ delay is built in to allow recovery from a temporary decrease.
} 
gain to better rank the constraints in $\mathrm{Beam}^{6}$.

\section{$5 \quad$ Experiments}

In this section we report on some first classification and prediction experiments with a prototypical Prolog implementation of MACCENT. Both classification and numeric prediction could in fact be considered as special cases of the task of learning from a conditional probability distribution $\tilde{p}(C \mid I)$ : with classification $\tilde{p}(C \mid I)$ only takes values 0 and 1 ; with numeric prediction there are only two classes $C$. We would like to stress that the full power of MACCENT should best be demonstrated on the general case of multi-class datasets where $\tilde{p}$ can take any real value between 0 and 1 (e.g. distribution of votes for more than two candidates in different districts). At present however, such data do not seem to be readily available.

First, we round off the running example with the application of MACCENT to the multi-class animals domain. With the hold-out stopping criterion suppressed, MACCENT found 13 constraints before the log-likelihood of Table 1 was reduced to 0 . The first four of these clausal constraints are, in order of discovery,

\begin{tabular}{|c|c|c|c|}
\hline$\langle C, \leftarrow Q\rangle$ & gain & $\lambda$ & $1 \mathrm{~h}$ \\
\hline$\langle$ reptile, $\leftarrow$ has_cov(scales $)$, habitat(land $)\rangle$ & 0.27 & 1.59 & -1.11 \\
$\langle$ fish, $\leftarrow$ not(has_legs $)$, not $($ hom $)\rangle$ & 0.23 & 1.56 & -0.88 \\
$\langle$ bird, $\leftarrow$ has_cov $($ feath $)\rangle$ & 0.18 & 1.59 & -0.70 \\
$\langle$ mammal, $\leftarrow$ not $($ has_cov $($ feath $)$ ), hom $\rangle$ & 0.18 & 1.59 & -0.52 \\
\hline
\end{tabular}

with the approximate gain, best $\lambda$, and log-likelihood of the sample in columns 2,3 , and 4 respectively.

\subsection{Classification}

When compared to other inductive logic programming classifiers, MACCENT has the advantage that it induces a conditional probability distribution that can directly be combined with other stochastic information sources. To illustrate this point we conducted a relatively small experiment with natural language data extracted from the Wall Street journal corpus [Marcus et al., 1993].

In this corpus the words are tagged with so-called Parts-of-Speech (POS). We grouped the tags into 16 classes, the most important of which are noun, adjective, verb, punctuation, determiner, pronoun, adverb. Next, based on frequency counts in the corpus, we set up a dictionary that, given a word $w$ assigns to each class pos a lexical probability $p_{\text {dict }}(p o s \mid w)$. For instance, $p_{\text {dict }}($ adjective $\mid$ cross $)=0.03, p_{\text {dict }}($ noun $\mid$ cross $)=0.69$, $p_{\text {dict }}($ verb $\mid$ cross $)=0.28$. Then we selected from the corpus the first 1000 occurrences of words, such as cross, that are ambiguous between exactly the three classes noun, adjective, and verb. We stored each word, with

\footnotetext{
${ }^{6}$ MACCENT mainly employs the average of the actual and the potential approximate gain.
}

its class label, and the tagged left and right context in a separate instance. As background knowledge we specified Prolog predicates left(Index, Limit, Pos), and right(Index, Limit, Pos) that check whether a certain tag Pos appears in the context of a word within distance Limit, where Index is the position the word in the sentence. In the language $\mathcal{L}$, with a size of order $10^{15}$, we allowed combinations of these two predicates, with $\mathrm{Limit}$ taking values $1,2,3,4,5$, and unlimited, and with $P o s$ instantiated to one of the 16 morphological categories. Finally, we ran MACCENT on this 3-class problem to construct a probability distribution $p_{\text {context }}(p o s \mid w)$ based on the context of the word.

Classifying words into the class with highest probability as predicted by the model we ran a 10 -fold crossvalidation based on a random partition over 1000 instances, and each time measured the performance in terms of classification accuracy. The model $p_{\text {context }}(p o s \mid w)$ induced by MACCENT achieved an average accuracy of $70.4 \%( \pm 4.8)$. The model $p_{\text {dict }}(p o s \mid w)$ based on the dictionary had a much better score of $81.2( \pm 3.0)$. The best score however was obtained from the combination of the two models $p_{\text {context }}(p o s \mid w) * p_{\text {dict }}(p o s \mid w)$ which achieved an accuracy of $85.4( \pm 3.0)$.

Though MACCENT by itself is likely to perform better in the presence of more sophisticated background knowledge, this initial experiment with POS-tagging demonstrates the (poor) output of MACCENT can successfully be combined with other stochastic information sources. For a more mature application of maximum entropy method to the general POS-tagging problem, we refer to [Ratnaparkhi, 1996].

\subsection{Numeric prediction}

Recall that one of the inputs to MACCENT is an empirical distribution $\tilde{p}(C \mid I)$. In the previous examples, $\tilde{p}$ took only the values 0 and 1 . Multi-class datasets where $\tilde{p}$ can take any real value between 0 and 1 , do not seem to be readily available. There are however enough tasks available that involve regression, the prediction of a numeric value. These values can always be projected onto a scale from 0 to 1 . With some abuse of the intuitions behind a conditional probability model, MACCENT can now interpret the rescaled values as estimates of the probability that the example belongs the single "positive" class. The inverse "unscaling" projection allows one to calculate predictions from the induced model. Like the Fors system [Karalič and Bratko, 1997], which incorporates linear regression techniques, MACCENT can then perform first order regression from positive data only.

We applied this technique to two benchmark domains well known in the inductive logic programming community: mutagenicity [Srinivasan et al., 1996], and mesh design [Dolšak and Muggleton, 1992]. 
In the first domain the task is to predict the log mutagenicity of molecules on the basis of structural information on the compounds. We used the full set of available background predicates and defined a language $\mathcal{L}$ with size order $10^{24}$. With a 10 -fold crossvalidation we obtained an average standard error of $1.38( \pm 0.18)$, with log-mutagenicity ranging from -3 to 5.39 .

The second domain requires a prediction about the coarseness of a mesh model used to approximate physical structures. For every edge of the structure one has to decide on the number, ranging from 1 to 17, of subedges on the basis of properties of the edge and of related edges. We focused our experiments on the 5 structure $(\mathrm{A}, \mathrm{B}, \mathrm{C}, \mathrm{D}, \mathrm{E})$ dataset. With a language $\mathcal{L}$, with size of order $10^{7}$, we tried both a 5 -fold leave-one-structure-out (mesh-xv5) and a random 10-fold crossvalidation (meshxv10). We calculated standard error of the estimate, and accuracy. For accuracy we rounded MACCENT's predictions to the nearest class. The table below summarizes results for MACCENT and ICL [De Raedt and Van Laer, 1995].

\begin{tabular}{|c|c|c|c|c|}
\hline & \multicolumn{2}{|c|}{ Accuracy } & \multicolumn{2}{c|}{ Standard error } \\
\hline Algorithm & mesh-xv5 & mesh-xv10 & mesh-xv5 & mesh-xv10 \\
\hline ICL & 0.50 & $0.70( \pm 0.05)$ & $2.82( \pm 1.19)$ & $1.84( \pm 1.03)$ \\
MACCENT & 0.25 & $0.44( \pm 0.11)$ & $2.83( \pm 0.42)$ & $1.54( \pm 0.33)$ \\
\hline
\end{tabular}

The above table shows roughly the same picture for mesh-xv5 and mesh-xv10: MACCENT scores a lot worse on accuracy, but comparable or even slightly better on standard error. We take this as an indication that the scaling technique indeed makes sense, and that MACCENT can be considered for solving regression tasks.

\section{Conclusion}

We have presented a framework for combining maximum entropy modeling with inductive logic programming techniques, and a number of approximations that led to the definition and implementation of the MACCENT algorithm. First experiments indicate the first order statistical modeling algorithm MACCENT may be useful for prediction, and for classification in cases where the induced model should be combined with other stochastic information sources.

\section{Acknowledgements}

Luc Dehaspe is supported by the ESPRIT IV project no. 20237 on Inductive Logic Programming II (ILP ${ }^{2}$ ). He would like to thank Walter Daelemans for his crucial pointer to the Maximum Entropy literature; Adam Berger, Vincent Della Pietra, Stephen Della Pietra, and Adwait Ratnaparkhi for their inspiring papers; Bojan Dolsak and Ashwin Srinivasan for the mesh and mutagenicity data; Hendrik Blockeel and Dirk Roose for mathematical assistance; Bart Demoen and Wim Van
Laer for their contributions to the implementation of Maccent; and finally, Luc De Raedt and Maurice Bruynooghe for their creation of a stimulating (logical) context for this research.

\section{References}

[Berger et al., 1996] A.L. Berger, V.J. Della Pietra, and S.A. Della Pietra. A maximum entropy approach to natural language processing. Computational Linguistics, 22(1):39-71, 1996.

[Bratko, 1986] I. Bratko. Prolog Programming for Artificial Intelligence. Addison-Wesley, 1986.

[Brown, 1959] D. Brown. A note on approximations to discrete probability distributions. Information and Control, 2:386-392, 1959.

[Darroch and Ratcliff, 1972] J. N. Darroch and D. Ratcliff. Generalized Iterative Scaling for Log-linear Models. Annals of Mathematical Statistics, (43):14701480, 1972.

[De Raedt and Džeroski, 1994] L. De Raedt and S. Džeroski. First order $j k$-clausal theories are PAClearnable. Artificial Intelligence, 70:375-392, 1994.

[De Raedt and Van Laer, 1995]

L. De Raedt and W. Van Laer. Inductive constraint logic. In Proceedings of the 5th Workshop on Algorithmic Learning Theory, volume 997 of Lecture Notes in Artificial Intelligence. Springer-Verlag, 1995.

[De Raedt, 1996] L. De Raedt. Induction in logic. In R.S. Michalski and Wnek J., editors, Proceedings of the 3rd International Workshop on Multistrategy Learning, pages 29-38, 1996.

[Dehaspe and De Raedt, 1996]

L. Dehaspe and L. De Raedt. DLAB: A declarative language bias formalism. In Proceedings of the International Symposium on Methodologies for Intelligent Systems (ISMIS96), volume 1079 of Lecture Notes in Artificial Intelligence, pages 613-622. Springer-Verlag, 1996.

[Della Pietra et al., 1995] S.A. Della Pietra, V.D. Della Pietra, and J. Lafferty. Inducing features of random fields. Technical Report CMU-CS-95-144, Carnegie-Mellon University, Pittsburgh, PA, 1995.

[Dolšak and Muggleton, 1992] B. Dolšak and S. Muggleton. The application of Inductive Logic Programming to finite element mesh design. In S. Muggleton, editor, Inductive logic programming, pages 453-472. Academic Press, 1992.

[Gull and Daniell, 1978] S.F. Gull and G.J. Daniell. Image Reconstruction from Incomplete and Noisy Data. Nature, 272:686, 1978. 
[Jaynes, 1990] E.T. Jaynes. Notes on present status and future prospects. In W.T Grandy and L.H. Schick, editors, Maximum Entropy and Bayesian Methods, pages 1-13. Kluwer Academic Publishers, 1990.

[Karalič and Bratko, 1997] A. Karalič and I. Bratko. First order regression. Machine Learning, 1997. To appear.

[Marcus et al., 1993] M.P. Marcus, B. Santorini, and M. A. Marcinkiewicz. Building a large annotated corpus of English: the Penn Treebank. Computational Linguistics, 19(2):313-330, 1993.

[Muggleton, 1997] S. Muggleton. Stochastic logic programs. Journal of Logic Programming, 1997. submitted.

[Plotkin, 1970] G. Plotkin. A note on inductive generalization. In Machine Intelligence, volume 5, pages 153-163. Edinburgh University Press, 1970.

[Pompe and Kononenko, 1995] U. Pompe and I. Kononenko. Naive bayesian classifier within ilp-r. In L. De Raedt, editor, Proceedings of the 5th International Workshop on Inductive Logic Programming, pages 417-436, 1995.

[Ratnaparkhi, 1996] A. Ratnaparkhi. A maximum entropy part-of-speech tagger. In Proceedings of the Empirical Methods in Natural Language Processing Conference. University of Pennsylvania, 1996.

[Srinivasan et al., 1996] A. Srinivasan, S.H. Muggleton, M.J.E. Sternberg, and R.D. King. Theories for mutagenicity: A study in first-order and feature-based induction. Artificial Intelligence, 85, 1996. 